\title{
Non-Obstetric Pain in Pregnancy
}

\author{
Dr Abdul Lalkhen ${ }^{1}$ and Dr Kate Grady ${ }^{2}$ \\ ${ }^{1}$ Specialist Registrar and ${ }^{2}$ Consultant in Anaesthesia and Pain Medicine, South Manchester University Hospital
}

\section{S U M M A RY P O I N T S}

- The hormonal and mechanical changes which result from pregnancy have the capacity to exacerbate existing chronic pain conditions as well as produce pain unique to this physiological and anatomical state.

- Pain in pregnancy is very common and can impact negatively on maternal satisfaction with the pregnancy.

- Management of pain requires a multidisciplinary, biopsychosocial approach. The possible presence of co-existing pathology and obstetric conditions must be borne in mind when assessing pain in pregnancy.

- Treatment of pain in pregnancy should focus on non-pharmacological interventions in the first instance with due consideration of the risk of medications to the mother, fetus and the course of the pregnancy.

\section{Introduction}

This review considers pain arising as a direct consequence of pregnancy or pre-existing pain which is exacerbated by pregnancy. The latter situation may arise where treatment has been curtailed because of its potential detrimental impact on the fetus. The review alerts the reader to the importance of having excluded obstetric conditions as a cause of pain and deals with the safe management of pain in pregnancy. Serious obstetric conditions such as placental abruption and uterine rupture present with acute pain and it is important that the assessment process begins with obstetric expertise to assess and ensure the well-being of mother and fetus. Further, the specific implication of treatment on the pregnancy must also be considered.

Non-obstetric pain arising from pregnancy or pre-existing pain exacerbated by pregnancy is caused by changes in maternal anatomy and changes in maternal hormonal levels.

\section{Changes in maternal anatomy}

The most obvious anatomical change is the presence of the gravid uterus. This can cause pain from increased joint forces and increased lumbar lordosis. Stretching of the anterior longitudinal ligament due to increased lordosis as well as stretching, weakness and separation of abdominal muscles may result in pain. Joint laxity (hormonal effect) affecting anterior and posterior longitudinal ligaments of the lumbar spine and causing spreading of the symphysis pubis and sacroiliac joints has been implicated in pregnancy related pelvic pain ${ }^{1}$. However this assertion has been challenged by a study using ultrasonography which demonstrated an association between the width of the symphysis pubis and pain at that site, irrespective of serum relaxin concentrations ${ }^{2}$.
There is a significant increase in the anterior tilt of the pelvis necessitating increased use of hip extensor abductors and ankle plantar flexors. Lower abdominal pain has been attributed to stretching of the round ligaments.

The main factors are therefore probably mechanical as a result of the alteration in posture required to carry the increasing mass in the abdomen. Hormonal alterations result in changes in the pelvic ligaments. The hormone responsible is unclear and despite relaxin being implicated in the genesis of musculoskeletal problems no correlation was found between relaxin concentrations and either the measured laxity of the wrist joints or the onset of pelvic pain ${ }^{3}$.

Stones and Vits ${ }^{4}$ conclude that muscular activity is required to maintain the stability of the joints of the spine and pelvis and thus may be compromised in the pregnant state leading to musculoskelelal pain.

\section{Back pain, pelvic girdle pain and symphysis pubis diastisis pain}

A prospective Swedish study found that $76 \%$ of women reported back pain at some point during their pregnancy ${ }^{5}$, whilst a prevalence of $3.9 \%$ to $89.9 \%$ (mean $45.3 \%$ ) was found by a group of authors ${ }^{6}$ who conducted a systematic review of studies which used the terms pregnancy related pelvic girdle pain and pregnancy related low back pain. The latter attributed the wide range of prevalence to variations in definition, classification and identification of 'lumbopelvic' pain. The unclear epidemiology of back pain and pelvic pain is further compounded by underreporting of back pain during pregnancy, particularly in the USA where it is regarded as a normal part of pregnancy. 
The prevalence of pelvic pain ranges from $16 \%$ to $20 \%$ with well conducted cohort studies demonstrating ongoing pelvic pain after pregnancy of $4-18 \%$ depending on the type of pelvic pain pattern diagnosed ${ }^{7}$. From a diagnostic and treatment point of view, tests to distinguish pelvic pain from back pain have been devised ${ }^{7,8}$. Average pain intensity during pregnancy was found to be $50 / 100 \mathrm{~mm}$ on a visual analogue scale with severe disability occurring in about $8 \%$ of patients 5 . The pain increases with advancing pregnancy and interferes with work, daily activities and sleep ${ }^{1,9}$. The exaggeration of the lumbar lordosis and increased joint laxity of pregnancy may cause spondylolisthesis. Pre-existing back pain can be exacerbated by pregnancy and it is important to exclude serious or sinister causes of back pain. Lumbovertebral disc abnormalities are not increased in prevalence in pregnant women, with nucleus pulposus herniation having an incidence of 1:10000. Whilst radicular symptoms often accompany back pain it is thought that this is due to a direct pressure effect of the fetal head on lumbosacral nerves ${ }^{10,11,12}$.

There is a paucity of well conducted randomised controlled trials with regard to the management of back and pelvic pain in pregnancy. There are no trials evaluating preventative strategies with regard to pregnancy related back and pelvic pain. Studies evaluating physiotherapy, water gymnastics, acupuncture and specialised pillows against usual perinatal care suffer from poor methods of randomisation, high attrition rates and inadequate allocation concealment. The nature of these interventions makes blinding almost impossible. A Cochrane review identified eight randomised controlled trials of therapeutic interventions ${ }^{9}$. The reviewers were unable to perform a meta-analysis due to the heterogeneity of the individual studies. They concluded that all the studies had a moderate or high potential for bias due to the factors mentioned above except for the paper by Elden evaluating acupuncture for pelvic pain ${ }^{13}$.

Recommendations with regard to the management of the problem of back and pelvic pain are difficult. The evidence suggests that pregnancy specific exercises are helpful and that the specially designed Ozzlo pillow which is no longer obtainable may relieve back pain. Water gymnastics may reduce back pain related absenteeism. Acupuncture resulted in more pain relief than physiotherapy however the acupuncture was delivered individually whilst the physiotherapy was group based. Treatment of symphysis pubis pain in the UK involves avoidance of abduction and other activities which induce pain as well as the use of a support belt. This is in contrast to an observational Dutch study which advocates exercise to increase the range of hip abduction and challenging functional limitations $s^{4,14}$. The authors of a review on the subject of pain in pregnancy describe their experience of a multidisciplinary team approach in terms of evaluation and treatment ${ }^{15}$. The delivery of appropriate biopsychosocial interventions with regard to back and pelvic pain was facilitated.

Back pain after delivery continues in $43 \%$ of sufferers at 6 months. The pain is worse in the younger multigravid parturient with a history of pre-pregnancy back pain ${ }^{17}$. Patients who have had intrapartum epidural analgesia may complain of a very localised back pain, simply attributable to the insertion of the epidural needle. This is often due to local trauma; however a high index of suspicion should be maintained for an epidural abscess.

There is high quality, conclusive evidence that after childbirth there are no differences in the incidence of long term low back pain between women who receive epidural pain relief and those who receive other forms of pain relief ${ }^{18,19}$.

\section{Nerve entrapment syndromes}

Evidence to support the treatment of nerve entrapment syndromes is based on pathophysiological mechanisms and not randomised controlled trials.

Stretching of the abdominal wall potentially causes nerve entrapment syndromes. The thoracic nerves pierce the rectus abdominis muscles and traction or entrapment of these may cause unilateral pain. Injection of local anaesthetic and steroid at the point of emergence of the nerve may be an effective treatment ${ }^{20}$.

A similar situation may arise with the iliohypogastric, genitofemoral and lateral cutaneous nerve of the thigh within the pelvis. Genitofemoral pressure can cause pain in the groin and labia and entrapment of the lateral cutaneous nerve of the thigh will cause pain in the lateral thigh (meralgia paraesthetica) $)^{21,22}$.

Fluid retention, fat deposition and the effect of relaxin on the transverse carpal ligaments makes carpal tunnel syndrome more common in pregnancy. Elevation of the limb and splinting devices are preferable to surgery, which should be reserved for severe $\operatorname{cases}^{23}$.

\section{Post-caesarean section incisional pain, perineal pain and dys- pareunia and coccydynia}

Following caesarean section, pains may be caused by incisional scars, neuromata or neuropathic pain deep to the incision and can be treated with infiltration of steroid and local anaesthetic, amitriptyline, lignocaine transdermal patches or surgical revision ${ }^{15}$. A Cochrane review of therapeutic ultrasound for these pains advocated caution with regard to the value of this treatment ${ }^{24}$.

Coccydynia in pregnancy is associated with ligamentous laxity and changes in posture and may be worse after labour. Pre-existing coccydynia may be exacerbated by pregnancy and delivery. A prospective trial in non-pregnant patients found that $60 \%$ of patients responded to infiltration of the area with local anaesthetics and steroids ${ }^{16}$. Heat packs and a ring cushion may be effective treatment modalities. There is a paucity of robust evidence for treatment of this condition. 


\section{Headache}

The commonest forms are tension headache or migraine with the usual pattern being improvement over time particularly towards the end of the first trimester. There is a tendency towards relapse after the third trimester. Management consists of recognising pre-eclampsia or imminent eclampsia, excluding focal lesions or subarachnoid haemorrhage, and excluding depression in the first instance. Nonpharmacological measures and simple analgesics form the mainstay of therapy ${ }^{15}$.

\section{Pain Management}

Management of the pregnant pain patient must be in the context of the multidisciplinary pain service liaising with the obstetric and midwifery team ${ }^{15}$. In practice, anaesthetists undertaking antenatal anaesthetic assessment clinics are often asked to advise.

Non-pharmacological management

These methods should be employed where possible in order to circumvent the potentially harmful effects of systemic analgesia on the fetus, the pregnancy and the woman ${ }^{15,25}$. Physiotherapy may be helpful by the provision of sacroiliac belts, back care classes and muscle relaxation techniques but the evidence for their effectiveness is poor. Physical therapy advice plays a major role in the management of pain in pregnancy which largely has a musculoskeletal component ${ }^{15,20}$.

TENS can be used in the management of back pain and nerve entrapment syndromes ${ }^{26}$. Placement of electrodes on the anterior abdominal wall should be avoided as this carries the risk of precipitating labour (with electricity having historically been used to induce labour ${ }^{27}$ ).

Acupuncture appears to be a safe form of analgesia and can be used for pelvic pain, headaches and back pain ${ }^{13}$; however, practitioners should be appropriately trained since one common method of abortion is simply to use strong acupuncture point stimulation of San Yin Chiao (SP6) in conjunction with He Gu (LI4). Furthermore, this is potentially a resource-intense modality of treatment and the evidence for its effectiveness needs to be more robust than currently exists.

\section{Psychosocial assessment}

Psychological distress as well as depression and anxiety can precipitate, maintain and exacerbate pain states. An exploration of the stressors to which the patient is exposed is advised. There should be awareness that domestic violence is significantly increased in pregnancy. A cognitive behavioural (CBT) based strategy which addresses these factors may avoid the use of unnecessary medication in patients with headaches in pregnancy ${ }^{15}$. Based on the evidence for CBT in chronic pain states and the nature of pain it may be tentatively suggested that there is a role for psychosocial evaluation at the very least and for CBT in certain cases.
Pharmacological treatment

Severe or chronic pain, if left untreated or inadequately treated, can have adverse effects on both the mother and the fetus. However, the most effective and safe drug regimen for treatment of pain during pregnancy remains controversial ${ }^{25}$.

In the UK approximately 1:40 babies will be born with a malformation. From conception to the first missed period, the embryo is thought to be resistant to any teratogenic effects of medicines. Embryogenesis starts at about 17 days post-conception and is complete by $60-70$ days. During this period exposure to certain medicines may result in major birth defects. Exposure following this period may not result in major defects; however some medicines can interfere with functional development of organ systems ${ }^{25,28}$.

\section{Paracetamol}

Paracetamol remains the analgesic of choice during pregnancy, although it should not be taken unnecessarily. Frequent use of paracetamol from 20-32 weeks has been associated with asthma, wheezing and elevated $\operatorname{IgE}$ in a subgroup of school aged children ${ }^{29,30}$. A causal link is assumed by the authors with an estimated $1 \%$ of childhood wheezing potentially attributable to paracetamol.

\section{Non-steroidal anti-inflammatory drugs}

NSAIDS effect renal and cerebral blood flow in the fetus (indeed the former is the rationale behind their occasional use in polyhydramnios) but there are concerns about causation of growth restriction. Ibuprofen and naproxen have not been linked to congenital defects when taken up to 30 weeks of pregnancy and are compatible with breastfeeding ${ }^{31,32}$. Chronic exposure or exposure to high doses after 30 weeks of pregnancy may be associated with bleeding complications and premature closure of the ductus arteriosus. Studies which demonstrate a statistical link between NSAID use and miscarriage are of poor quality in terms of defining exposure and the statistical association does not imply causation. In general, it is advisable to avoid the use of NSAIDS for analgesia in pregnancy.

\section{Opioids}

Dependence and withdrawal symptoms may manifest in the neonate from chronic maternal opioid use. In the Boston Collaborative Perinatal Project study there was no significant increase overall in either major or minor malformations with codeine. Although there was a slight, but statistically significant, increase in respiratory tract malformations following first trimester use ${ }^{33}$. Occasional therapeutic use, however, based on the available evidence, is not likely to cause fetal damage.

The limited data on the use of dihydrocodeine in human pregnancy do not indicate an increased risk of fetal toxicity.

There are no published data on the outcome of human pregnancy after tramadol exposure in the first trimester. Tramadol has been used during labour with no significant adverse effects on the fetus/neonate.

Overall there is no good evidence that the use of therapeutic doses in pregnancy have an adverse effect on fetal development. Opioids 
may be used at any stage of pregnancy for the short term treatment of moderate to severe pain ${ }^{15,25,33}$.

The weak opioid analgesic of choice in pregnancy is codeine, as there is more documented experience with its use. It should be borne in mind that most pregnant women are constipated and this may be exacerbated by codeine. For severe pain, morphine is the treatment of choice: the main concern is the risk of neonatal withdrawal and respiratory depression of the newborn ${ }^{33}$.

\section{Local anaesthetics}

In 293 children borne of women who were exposed to lidocaine in the first four months of pregnancy the Collaborative Perinatal Project demonstrated no increase in the frequencies of congenital anomalies in general, of major malformations, of minor anomalies, and of major classes of congenital anomalies ${ }^{33}$.

There is no available data on the use of lidocaine transdermal patches in pregnancy. No accumulation of lidocaine in plasma was observed in healthy volunteers after repeated application of the maximum recommended dose.

\section{Tricyclic antidepressants}

The information from the National Teratology Information Service states that epidemiological studies have shown no evidence that therapeutic doses of tricyclic antidepressants are associated with an increased incidence of birth defects. Chronic use, or the use of high doses near term, has been associated with neonatal withdrawal symptoms in some cases. If amitriptyline is required throughout pregnancy it is recommended that the dose is tapered 3-4 weeks prior to delivery to reduce the likelihood of neonatal withdrawal symptoms.

Amitriptyline and imipramine are the recommended drugs of choice for the treatment of depression during pregnancy, based on the length of time that they have been in use and the cumulative data on their lack of fetotoxicity.

Capsaicin

The safety of capsaicin during pregnancy or lactation has not been established in either humans or animals. However, with the small amounts absorbed transdermally from capsaicin cream, it is considered unlikely that capsaicin will cause any adverse effects in humans.

\section{Gabapentin and Pregabalin}

There is no evidence of impaired fertility or harm to the fetus from gabapentin administration in animal studies. Evidence from literature reports are conflicting with the Gabapentin Pregnancy Registry recording congenital malformations similar to those reported in women with epilepsy and in the general population ${ }^{34}$. Gabapentin is not recommended for use in human pregnancy by the manufacturers.

Pregabalin use at any stage in pregnancy is also not recommended due to the lack of available human data and reports from animal studies of harm to the fetus. In animal studies there have been reports of skeletal abnormalities and neural tube defects. The National Teratology Information Service has not produced written recommendations but does not advise termination of pregnancy if a patient has had an exposure to pregabalin in pregnancy. The use of detailed ultrasound scans should be available.

\section{Pharmacological summary}

An analgesic ladder in pregnancy would start with paracetamol which remains the analgesic of choice. Codeine would be next in line in combination with paracetamol. Severe pain would be treated with morphine. Strong opioids do not appear to present an increased risk of fetal abnormalities; however neonatal withdrawal and respiratory depression may be a problem. Amitriptyline would be the drug of choice for neuropathic pain.

\section{Acknowledgements}

Thanks to Mr Charles Cox, Consultant Obstetrician and Gynaecologist for his advice on this manuscript.

\section{REFERENCES}

1. MacEvilly M, Buggy D. Back pain and pregnancy: a review. Pain 1996; 64: 405-414

2. Marnach ML, Ramin KD, Ramsey PS, et al. Characterization of the relationship between joint laxity and maternal hormones in pregnancy. Obstet Gynecol 2003; 101: 331-335

3. Bjorklund K, Bergstrom S, Nordstrom ML, et al. Symphyseal distention in relation to serum relaxin levels and pelvic pain in pregnancy. Acta Obstet Gynecol Scand 2000; 79: 269-275

4. Stones RW, Vits K.. Pelvic girdle pain in pregnancy Brit Med J 2005; 331: 249-250

5. Kristiansson P, Svardsudd K, von Schoultz B. Back pain during pregnancy: a prospective study. Spine 1996; 21(6): 702-709

6. Wu WH, Meijer OG, Uegaki K, et al. Pregnancy-related pelvic girdle pain. I. Terminology, clinical presentation, and prevalence. Eur Spine J 2004; 13: 575-589

7. Albert H, Godskesen M, Westergaard J. Prognosis in four syndromes of pregnancy-related pelvic pain. Acta Obstetricia et Gynecologica Scandinavica 2001; 80: 505-510

8. Ostgaard HC, Zetherstrom G, RoosHansson E. Back pain in relation to pregnancy: a 6-year follow-up. Spine 1997; 22: 29452950 
9. Pennick VE, Young G. Interventions for preventing and treating pelvic and back pain in pregnancy. Cochrane Database of Systematic Reviews 2007, Issue 2. Art. No.: CD001139. DOI: 10.1002/14651858.CD001139.pub2

10. LaBan MM, Perrin JCS, Latimer FR. Pregnancy and the herniated lumbar disc. Arch Phys Med Rehab 1983; 64: 319-321

11. Weinreb TC. Wolbarsht LB, Cohen TM, et al. Prevalence of lumbosacral intervertebral disc abnormalities in MR images of pregnant and asymptomatic non pregnant women. Radiology $1989 ; 170: 125-128$

12. Fast A, Shapiro D, Ducommun EJ, et al. Low back pain in pregnancy. Spine 1987; 12: 368-371

13. Elden H, Ladfors L, Olsen MF, et al. Effects of acupuncture and stabilising exercises as adjunct to standard treatment in pregnant women with pelvic girdle pain: randomised single blind controlled trial. Brit Med J 2005; 330: 761

14. Röst C. Bekkenpijn tijden en na de Zwangerschap: Een Programma ter Voorkoming van Chronische Bekkeninstabiliteit. Maarssen: Elsevier, De Tijdstroom, 1999

15. Roche S, Hughes EW. Management of pain problems in pregnancy. Pain Reviews 1999; 6: 239-261

16. Wray C, Easom S, Hoskinson J. Coccydynia; aetiology and treatment. J Bone Joint Surg Br 1991; 73B: 335-338

17. Turgut F, Turgut M, Cetinsahin M. A prospective study of persistent back pain after pregnancy. Eur J Obstet Gynecol Reprod Biol 1998; 80: 45-48

18. Howell CJ, Dean T, Lucking L, et al. Randomised study of long term outcome after epidural versus non-epidural analgesia during labour. Brit Med J 2002; 325: 357-358

19. Loughnan BA, Carli F, Romney M, et al. Epidural analgesia and backache: a randomized controlled comparison with intramuscular meperidine for analgesia during labour Br. J. Anaesth 2002; 89: 466-472

20. Peleg R, Gohar J, Koretz M, et al. Abdominal wall pain in pregnant women caused by thoracic lateral cutaneous nerve entrapment. Eur J Obstet Gynecol Reprod Biol 1997; 74: 169-171

21. Vargo M, Robinson L, Nicholas J, et al. Postpartum femoral neuropathy: relic of an earlier era? Arch Phys Med Rehabil 1990; 71: 591-596

22. Deal C, Canoso J. Meralgia paraesthesia and large abdomens. Ann Intern Med 1982; 96: 787-788
23. Seror P. Pregnancy-related carpal tunnel syndrome. J Hand Surg [Br] 1998; 23: 98-101

24. Hay-Smith EJC.Therapeutic ultrasound for postpartum perineal pain and dyspareunia. Cochrane Database of Systematic Reviews 1998, Issue 3. Art. No.: CD000495. DOI: 10.1002/14651858. CD000495

25. Rathmell JP, Viscomi CM, Ashburn MA. Pain management during pregnancy. Anesth Analg 1997; 85: 1074-1087

26. Fisher A, Hanna M. Transcutaneous electrical nerve stimulation in meralgia paraesthetica of pregnancy. Br J Obstet Gynaecol 1987; 94: 603-605

27. Theobold GW, Lundbord RA. The electrical induction of labour with the transitor pulse generator and pulse current measuring device. Br J Obstet Gynaecol 1962; 69(3): 434-442

28. Prescribing Medicines in Pregnancy. An Australian categorisation of risk of drug use in pregnancy 1999 http://www.tga.gov.au/docs/pdf/medpreg.pdf

29. Shaheen SO, Newson RB, Sherriff A, et al. Paracetamol use in pregnancy and wheezing in early childhood. Thorax 2002; 57: 958-963

30. Shaheen SO,Newson RB, Henderson AJ, et al. Prenatal paracetamol exposure and risk of asthma and elevated Immunoglobulin $\mathrm{E}$ in childhood. Clin Exp Allergy 2005; 35: 18-25

31. Briggs GG, Freeman RK, Yaffe SJ. Drugs in pregnancy and lactation. Baltimore: Williams \& Wilkins, 1990

32. American Academy of Pediatrics Committee on Drugs. The transfer of drugs and other chemicals into human milk. Paediatrics 1994; 93:137-150

33. Heinomen OP, Slone S, Shapiro S. Birth defects and drugs in pregnancy. Littleton, MA: Publishing Science Group, 1977

34. Montouris G. Gabapentin exposure in human pregnancy: results from the Gabapentin Pregnancy Registry. Epilepsy Behav 2003; 4; 310-317

AG Lalkhen FRCA D.P.Med (CARCSI), Specialist Registrar Anaesthesia and Pain Medicine

K Grady FRCA, Consultant in Anaesthesia and Pain Management, Department of Anaesthesia, South Manchester University Hospital, Southmoor Road, Manchester M23 9LT

Correspondence to: alalkhen@doctors.org.uk 